
\title{
3 Researchguare
}

\section{The apelin-13 peptide protects the heart against apoptosis through the ERK/MAPK and PI3K/AKT signaling pathways.}

\section{Xuejun Wang}

Jiangsu Province Hospital and Nanjing Medical University First Affiliated Hospital

\section{Li Zhang}

Jiangsu Province Hospital and Nanjing Medical University First Affiliated Hospital

\section{Mengwen Feng}

Jiangsu Province Hospital and Nanjing Medical University First Affiliated Hospital

Hao Zhang

Jiangsu Province Hospital and Nanjing Medical University First Affiliated Hospital

Jia Xu

Jiangsu Province Hospital and Nanjing Medical University First Affiliated Hospital

\section{Zijie Cheng}

Jiangsu Province Hospital and Nanjing Medical University First Affiliated Hospital

Lingmei Qian ( $\square$ Imqian@njmu.edu.cn )

Jiangsu Province Hospital and Nanjing Medical University First Affiliated Hospital

https://orcid.org/0000-0002-5603-3886

\section{Research article}

Keywords: Apelin-13, Doxorubicin, Heart failure, Oxidative stress, Apoptosis

Posted Date: September 4th, 2020

DOI: https://doi.org/10.21203/rs.3.rs-42016/v2

License: (c) (1) This work is licensed under a Creative Commons Attribution 4.0 International License.

Read Full License 


\section{Abstract}

Background: It has been acknowledged that endocrine activity is associated with the function of multiple systems in vivo. The apelin-13 peptide has been demonstrated to play a crucial role in physiological and pathological processes. However, the function of apelin-13 peptide in doxorubicin (DOX)-induced cardiotoxicity is unknown.

Methods: We explored the function and mechanism of apelin-13 peptide in apoptosis and oxidative stress by cell counting kit-8 (CCK-8) assay, trypan blue staining, TUNEL, lactate dehydrogenase (LDH), mitochondrial membrane potential assay kit with JC-1 (JC-1) and western blot in vitro. Then we verified the effect of apelin-13 in vivo by detecting serum apelin-13, CKMB, LDH, cardiac troponin I (cTnI) and cardiac troponin T (cTnT). EF, FS and LVEDs were used to identify the structural modification by echocardiography. Sirius red staining and HE staining assay were used to detecting the myocardial fibers alteration under apelin-13 treatment.

Results: Treatment with apelin-13 peptide significantly enhanced cell viability, mitochondrial membrane potential, but reduced LDH release, rate of apoptotic cells and activation of caspase-3 in vitro. In mice, apelin-13 alleviated the heart dysfunction induced by DOX. 4-oxo-6-((pyrimidin-2-ylthio)methyl)-4H-pyran3-yl 4-nitrobenzoate (ML221) inhibited the effect of extracellular signal-related kinases (ERK), phosphatidylinositol 3 kinases (PI3K) and protein kinase B (AKT) proteins phosphorylation expression compared with DOX.

Conclusion: The apelin-13 and apelin receptor (APJ) interaction on the cell membrane inhibits apoptosis through the ERK/mitogen-activated protein kinase (MAPK) and PI3K/AKT signaling pathways. Our research gives a first glimpse on the biological function and mechanism of apelin-13 on cardiotoxicity.

\section{Introduction}

Doxorubicin (DOX) has been identified as one of the most common treatments for tumors[1]. Chemotherapeutics could lead to severe side effects, most common of which is cardiotoxicity[2]. Mounting shreds of evidence manifested that long-term use of DOX could lead to arrhythmias and even to heart failure[3]. Because of its cardiac toxicity, the clinical application is severely limited. For decades, cardiotoxicity caused by anthracyclines through oxidative stress is one of the most acceptable theories at present[4]. However, the biomolecular mechanism of DOX in cardiomyocytes needs further to be elucidated.

Oxidative stress and apoptosis are crucial important pathogenesis of cardiotoxicity. Oxidative stress could mainly induce apoptosis through mitochondrial dysfunction, death receptor pathway and endoplasmic reticulum stress. When cells are stimulated by oxidative stress, the accumulation of oxidizing substances, such as free radicals, causes damage to organelles, leading to cell death[5]. Recent studies have demonstrated that apoptosis and ROS are associated with cellular damage incurred during oxidative stress[6]. Oxygen-free radicals resulting from oxidative stress can be prevented[7], and targeting 
regulated cell death pathways before oxidative stress manifestation can alleviate oxidative impairment and potentially pave the way for new therapeutic plans for use of oxidative stress[8]. Oxidative stress has been identified as the major pathogenesis of DOX-induced cardiotoxicity[9]. To some extent, ROS generated by DOX could significantly increase mitochondrial oxidative stress, thus activate the activity of caspase-3 and other molecules associated with apoptosis[10]. Hence, inhibition of oxidative stress can not only reduce apoptosis but also may reduce the cardiotoxicity induced by DOX.

It is well known that peptides produced by proteasome degradation play important protective roles in various diseases and act as endogenous ligands or receptors to mediate varieties of signaling pathways[11]. For example, endorphins are endogenous peptides produced by the body that have similar effects as morphine and analgesics[12]. Cecropins play important roles in the antibacterial activity of Cterminal amides[13]. Angiotensin II binds to angiotensin receptors in heart and kidney to regulate water and salt balance and blood pressure[14]. Furthermore, the levels of natriuretic peptide family members are measured in the diagnosis of heart failure[15]. These peptides play indispensable roles in regulating physiological function and repairing human diseases. Apelin gene is located on the Q25-26.1 band of the $X$ chromosome which is expressed in vascular endothelial cells[16]. A small molecular peptide encoded by a gene and cut by a proteasome, preproapelin is composed of 77 amino acid residues and is decomposed to produce endogenous fragments such as apelin-36, apelin-13 and apelin-17[17]. Different subtypes of apelin are involved in different metabolic pathways. Studies have reported that apelin-36, apelin-17 and apelin-13 are active forms of apelin. Apelin-36 possesses a protective effect on MPPinduced neurotoxicity through PI3K/AKT/mTOR autophagy pathway[18]. And plasma apelin-17 levels were positively correlated with collateral circulation enhancement in patients with ischemic stroke, which might be mediated by NO-cGMP pathway[19]. The receptor for the apelin receptor early endogenous ligand (APELA) and apelin (APLN) hormones is named APLNR or APJ and coupled to $G$ proteins that inhibit adenylate cyclase activity[20]. Studies have reported that the receptor for the APELA hormone, APJ[21], plays a pivotal role in early development.

Researches have demonstrated that apelin-13 has potency in lowering blood pressure and modulating heart development[22]. Given the protective effect of apelin-13 in cardiovascular system, we investigated its role in cardiotoxicity. Meanwhile, the biomolecular mechanism of DOX-induced cardiotoxicity still has not been clarified. Our study suggested that apelin-13 bestowed cardioprotective effects against DOXinduced cardiotoxicity by inhibiting apoptosis and oxidative stress through ERK/MAPK and PI3K/AKT signaling pathway. $\mathrm{CoCl}_{2}$ can also elicit cardiac complications by inducing oxidative stress and apoptosis. We also provide evidence that apelin-13 binds to APJ to attenuate apoptosis and oxidative stress. In summary, our results shed light on the role of apelin-13 during the molecular regulation of apoptosis and oxidative stress and provided a novel interventional treatment for cardiovascular disease.

\section{Materials And Methods}

\subsection{Reagents and antibodies}


Doxorubicin and cobalt chloride were purchased from MedChemExpress (New Jersey, America) and Sigma, respectively. Primary antibodies for the following proteins were purchased from cell signaling technology (Danvers, MA): $\beta$-actin (1:5000), total-PARP (1:1000), total caspase-3 (1:1000), total ERK1/2 (1:1000), phosphorylated ERK1/2 (1:1000), total PI3K (1:1000), phosphorylated PI3K (1:1000) and total AKT (1:1000), phosphorylated AKT (1:1000). Besides, anti-rabbit IgG and HRP-linked antibody were purchased from Biosharp Life Science (Beijing, China). Mitochondrial membrane potential assay kits with JC-1 and a LDH cytotoxicity assay kit, relative oxygen species assay kit, Cell Counting Kit-8, Trypan blue staining cell viability assay kit and one-step TdT-mediated dUTP nick end-labeling apoptosis assay kit were obtained from Beyotime Biotechnology (Shanghai, China). ML221 was purchased from MedChemExpress (New Jersey, United States).

\subsection{Peptide synthesis and administration}

Apelin-13 CMPLHSRVPFP peptide and scramble MPCLSHPVFPR peptide were synthesized with purity $>95 \%$ by Shanghai Science Peptide Biological Technology Co. LTD. (Shanghai, China). The peptide powder was dissolved in sterile water to generate a $10 \mu \mathrm{M}$ stock solution and was diluted to the experimental concentration.

\subsection{Cell culture}

Rat primary cardiomyocytes were extracted from rat one-half day after birth. The blood, fat and connective tissues were separated from heart tissues and digested with trypsin at $37^{\circ} \mathrm{C}$ at $60 \mathrm{rpm}$ for 15 min. The solution was removed and repeated the digestion three times. The cell-containing digestive fluids were placed in a centrifuge tube and centrifuged together after passing through 180 mesh. Cells were suspended in $10 \mathrm{ml}$ DMEM containing 10\% horse serum (HS, GIBCO, USA) and incubated in a 10 $\mathrm{cm}^{2}$ dish for $1.5 \mathrm{~h}$. The cell suspension was removed, and the cells $\left(5 \sim 6 \times 10^{5}\right.$ cells per dish) were inoculated into a new plate as previously described.

\subsection{Animals}

Male C57BL/6J mice (6-10 weeks of age, 20 22 g) were obtained from the Model Animal Research Center of Nanjing University (Nanjing, Jiangsu, China) and all procedures were followed in accordance with the ethical committee of Nanjing Medical University. All animals were raised at $20 \sim 25^{\circ} \mathrm{C}$ and in 50 70\% relative humidity. These mice were randomly divided into four groups (scramble peptide treated group, apelin-13 treated group, DOX and scramble peptide co-treatment group and DOX and apelin-13 cotreatment group) and 6 mice per group. The mice were treated with $5 \mathrm{mg} / \mathrm{kg} /$ week DOX i.p for five consecutive weeks and $1.5 \mu \mathrm{mol} / \mathrm{kg}$ apelin-13 were administrated daily i.p. Electrocardiograms were obtained 6 weeks later and the mice were sacrificed. The mice were treated according to the experimental requirements. All animal experiments complied with the Guide for the Care and Use of Laboratory Animals published by the National Institutes of Health (NIH Publications No. 85-23, revised 1996).

\subsection{Cell death rates and CCK-8 assay}


Trypan blue staining was used to calculate the mortality of rat primary cardiomyocytes. The cell mortality rate was obtained by counting the stained ones. The cells were collected at different lengths of times ( 0 , $6,12,18,24$ and $36 \mathrm{~h}$ ) and stained by a trypan blue staining cell viability assay kit to determine the cell death rate. At different concentrations of $\operatorname{DOX}(0.1,0.5,1.0,2.0$ and $5.0 \mu \mathrm{M})$ and $\mathrm{CoCl}_{2}(200,400,600,800$ and $1000 \mu \mathrm{M}$ ) were measured according to the manufacturer's instructions. CCK-8 assay can be used to evaluate cell proliferation and viability. The absorbance of different groups was measured at $450 \mathrm{~nm}$ and the cell survival rates were calculated according to the manufacturer's instructions.

\subsection{Lactate dehydrogenase (LDH) level detection}

Levels of lactate dehydrogenase $(\mathrm{LDH})$ released were detected in serum using an LDH release assay kit according to the manufacturer's protocol.

\subsection{JC-1 assay}

The mitochondrial membrane potential was measured by a mitochondrial membrane potential assay kit with JC-1 according to the manufacturer's instructions. The cells were cultured in serum-free DMEM containing (1x) JC-1 staining working fluid at $37^{\circ} \mathrm{C}$ for $20 \mathrm{~min}$. Then, washed twice with $\mathrm{JC}-1$ buffer and the cells were photographed by a fluorescence microscope (BX61; Olympus Corporation, Tokyo, Japan) after added $2 \mathrm{ml}$ DMEM. The JC-1 density was assessed by Image J software and calculated upon normalization to the control.

\subsection{TUNEL staining assay}

The rate of cell apoptosis can be detected by TUNEL assay. Cells were seeded ( $1 \times 10^{5}$ cells per well) in 6well dishes. Then the cells were washed once with phosphate-buffered saline (PBS) and fixed with $4 \%$ paraformaldehyde. Apoptotic cells were visualized with stained cells according to the manufacturer's protocol. TUNEL fluorescence intensity/DAPI fluorescence density was used to calculating the percentage of positive cells and the density was evaluated using ImageJ software 1.26 (Wayne Rasband, National Institutes of Health, Bethesda, MD, USA).

\subsection{Echocardiography}

Male C57BL/6J mice were anesthetized with isoflurane $(3 \%$ in $2 \mathrm{~L} / \mathrm{min})$, then they were placed on a heated animal operating table to maintain at $36.5 \sim 37.5^{\circ} \mathrm{C} \otimes$ which was monitored with a YSI telethermometer. Unhaired and detected ejection fraction (EF), fractional shortening (FS) and left ventricular end-systolic diameter (LVEDs) by echocardiography (vevo3100, FUJIFILM VisualSonics) respectively. All animal experiments complied with the Guide for the Care and Use of Laboratory Animals published by the National Institutes of Health (NIH Publications No. 85-23, revised 1996).

\subsection{Enzyme linked immunosorbent (ELISA) assay}


Serum CKMB, cTnl, cTnT and apelin-13 level were determined by commercially available ELISA kit (Mlbio, Shanghai, China) according to the manufacturer's instructions.

\subsection{Western blot analysis}

Proteins were isolated from cells using lysis buffer (containing Radio-Immunoprecipitation Assay (RIPA) and $1 \%$ Phenylmethylsulfonyl fluoride (PMSF)). Protein quantification was performed using a BCA protein detection kit (23229; Thermo Fisher Scientific). Protein samples of the same mass were separated on $10 \%$ SDS-PAGE gels and transferred to nitrocellulose membranes (Millipore, Billerica, MA, USA), blocked with $5 \%$ skim milk for $1 \mathrm{~h}$ and then incubated with specific primary antibodies. A FluorChem $\mathrm{M}$ system was used to quantify the positive bands representing proteins involved in the orchestrated immune responses (ProteinSimple, San Jose, CA, USA).

\subsection{Statistical analysis}

All results are expressed as the mean $\pm S D$. Student's t-tests were used within two groups to determine significant differences. Comparisons between multiple groups were performed by one-way analysis of variance (ANOVA), and $\left.\left.p<0.05{ }^{(}\right), p<0.01{ }^{(* \star}\right)$ and $\left.p<0.001{ }^{(* *}\right)$ were considered significant. All experiments were repeated at least three times unless otherwise specified. All data were analyzed by GraphPad Prism software.

\section{Results}

\subsection{Sequences of apelin-13 and its family.}

Apelin, the endogenous ligand of APJ, to be discovered expressed widely in cardiovascular system. As a vasoactive peptide, it could interact with APJ receptor to regulate cardiovascular physiology and pathophysiology. Apelin-13, -17 and -36 are active isoforms of apelin, which were derived from apelin-77 (pre-pro-apelin). Apelin-77 is modified by endopeptidase to form pro-apelin-55, which is further cleaved to apelin-36, -17,-13 and etc. Different apelin subtypes have different functions, however, apelin 13 and [Pyr]-Apelin-13 are considered to be more dominant and potent isoforms in cardiovascular system. We found that apelin and its family are named according to the length of their sequence (Fig. 1). However, the biological function and mechanism still need to be clarified.

\subsection{The effect of doxorubicin and cobalt chloride on cell viability and death.}

To study the function of apelin-13 in oxidative stress, we established a DOX-induced cardiotoxicity model and $\mathrm{CoCl}_{2}$-induced hypoxia model. The cell mortality rate of DOX began to increase at $1.0 \mu \mathrm{M}$ for $12 \mathrm{~h}$ (Fig. 2A). Similarly, 1.0, 2.0 and 5.0 MM DOX reduced cell survival index equivalently (Fig. 2B). Then the rat primary cardiomyocytes were incubated for $0,6,12$ and $24 \mathrm{~h}$ with $1 \mu \mathrm{M} \mathrm{DOX}$, we found that $1 \mu \mathrm{M} \mathrm{DOX}$ cultured for $12 \mathrm{~h}$ or $24 \mathrm{~h}$ both increased cell death rate and reduced cell viability significantly (Fig. $2 \mathrm{C}$ and 
2D). As shown in Fig.2E, the result indicated that $800 \mu \mathrm{M}$ and $1000 \mu \mathrm{M}$ treatment both reduced cell viability significantly for $24 \mathrm{~h}$. Then trypan blue assay was used to identify $800 \mu \mathrm{M} \mathrm{CoCl}_{2}$ for different durations. Our results demonstrated that cell viability significantly began to reduce after 18h (Fig. 2F). Thus, we constructed DOX and $\mathrm{CoCl}_{2}$ models for further study successfully.

\subsection{Apelin-13 inhibits apoptosis by attenuating doxorubicin-induced cardiotoxicity.}

To figure out the biological function of apelin-13 in DOX-induced cardiotoxicity in rat cardiomyocytes, pretreatment with apelin-13 at different concentrations $(0.1,0.5,1.0,2.0$ and $5.0 \mu \mathrm{M})$ for $1 \mathrm{~h}$ and $1 \mu \mathrm{M}$ apelin-13 dramatically reduced the mortality of DOX-treated primary cardiomyocytes (Fig. 3A). There was no difference between each group in trypan blue assay, thereby scramble peptide is inactive (Fig.3B). DOX-induced cardiotoxicity declined cell viability significantly, but apelin-13 inhibited the effect and increased cell survival rate (Fig. $3 \mathrm{C}$ ). The increased cell death led to destruction of cell membrane, which was measured by LDH release assay. Apelin-13 significantly reduced LDH release compared with DOX (Fig. 3D). The western blot result revealed that the cleaved caspase 3 and PARP were activated by DOX and apelin-13 reduced the elevated expression of cleaved caspase-3 and PARP compared with DOX (Fig. $3 E$ ). Next, we found that the number of apoptotic cells was increased in the DOX-induced group but reduced after apelin-13 co-treatment (Fig. 3F). The increased formation of JC-1 monomer reflects the elevated mitochondrial membrane potential, indicating that DOX promoted apoptosis at the early state. Apelin-13 reduced the number of JC-1 monomers formation compared with DOX treatment (Fig. 3G). Therefore, our results indicated that apelin-13 possessed a protective role in DOX-induced cardiotoxicity.

\subsection{The effect of apelin-13 in $\mathrm{CoCl}_{2}$ induced oxidative stress.}

To test whether apelin-13 possessed a protective function in oxidative stress, we performed a comprehensive functional analysis. Trypan blue assay result demonstrated that the treatment of apelin13 decreased cell death rates compared with $\mathrm{CoCl}_{2}$ treated group (Fig. 4A). Then, Apelin-13 increased cell

viability compared with scramble peptide under $\mathrm{CoCl}_{2}$ treatment as evidenced by CCK-8 (Fig. 4B). We also proved that apelin-13 significantly decreased LDH release compared with $\mathrm{CoCl}_{2}$ (Fig. 4C). Furthermore, our result revealed that $\mathrm{CoCl}_{2}$ increased apoptotic cell rates, which was alleviated by apelin-13 (Fig. 4D), which was quantified by Fig. 4E. The expression of cleaved caspase-3 and PARP proteins were decreased by apelin- 13 treatment compared with $\mathrm{CoCl}_{2}$ (Fig. 4F). These findings suggested that apelin-13 reduced the apoptosis and cell damage induced by $\mathrm{CoCl}_{2}$.

\subsection{Apelin-13 significantly resists DOX-induced cardiotoxicity in vivo.}

Next, we tested the protective role of apelin-13 on rodent hearts in vivo. Male C57BL/6J mice were intraperitoneally injected with doxorubicin $(5 \mathrm{mg} / \mathrm{kg} /$ week) for five weeks consecutively and apelin-13 was injected daily i.p. Then they were subjected to echocardiography and sacrificed the following week (Fig. $5 A)$. Comparison with the heart rate of the four groups, we found that the heart rate was increased from $355.44 \pm 17.37 \mathrm{bpm}$ to $361.56 \pm 11.05 \mathrm{bpm}$ with apelin-13 treatment but then decreased to $282.11 \pm 10.52$ 
bpm in DOX model group. Apelin-13 effectively increased heart rate to $317.33 \pm 8.76 \mathrm{bpm}$ under DOX treatment (Fig. 5B). In view of the short half-life of apelin-13, we compared the levels of serum apelin -13 in each group before and after injection. We found apelin-13 level almost remained at the same level before administration. After 6 weeks of treatment, serum apelin-13 was decreased in DOX injected group compared with scramble peptide. Furthermore, serum apelin-13 level was increased significantly with apelin-13 and DOX co-treatment but inferior to the mice injected with apelin-13 only (Fig. 5C). The release of CKMB and LDH in peripheral blood were increased significantly in DOX treated mice and apelin-13 reduced the effect of DOX significantly (Fig. 5D). The levels of serum CTnI and CTnT were upregulated significantly when exposed to DOX. Co-treatment of apelin-13 downregulated serum cTnl and cTnT significantly compared with DOX treated mice (Fig. 5E). We also provided evidence that EF and FS rates were increased, whereas the LVEDs was decreased significantly by echocardiography after treated with apelin-13 (Fig. 5F). The evidence from Sirius red staining suggested that cardiac fibrosis was significantly reduced by apelin-13 compared with DOX (Fig. 5G). Accordingly, HE staining showed that apelin-13 attenuated the collagenous fiber rupture compared with DOX (Fig. 5H). Therefore, these results suggested that apelin-13 ameliorated the cardiotoxicity induced by DOX in mice.

\subsection{Apelin-13 inhibits cardiotoxicity through activating ERK/MAPK and PI3K/AKT signaling pathways.}

ERK/MAPK and PI3K/AKT are important pathways that protect the physiological function of the heart by inhibiting apoptosis. To verify the molecular mechanism by which apelin-13 protects primary cardiomyocytes against apoptosis, western blot analyses were performed. The result showed the expression of the phosphorylated ERK protein was activated upon apelin-13 treatment compared with DOX (Fig. 6A). Apelin-13 activated the expression of the phosphorylated AKT and PI3K proteins compared with DOX (Fig. 6B). When the action of $\mathrm{CoCl}_{2}$ was blunted, the expression of the phosphorylated ERK protein was activated by apelin-13 (Fig. 6C). Meanwhile, apelin-13 activated the expression of both phosphorylated proteins with $\mathrm{CoCl}_{2}$ treatment (Fig. 6D). These results revealed that apelin-13 protected cardiomyocytes from apoptosis through ERK/MAPK and PI3K/AKT signaling pathways.

\subsection{Apelin-13 protects cardiac function by binding APJ.}

To determine the role of ML221, we performed western blot to detect its interaction with apelin-13. We found ML221 inhibited the effect of apelin-13 on caspase-3 and PARP expressions of cardiomyocytes with DOX administration (Fig. 7A). As shown in our results, ML221 significantly inhibited the expression of the phosphorylated ERK protein with DOX and scramble peptide treatment, but activated ERK phosphorylation with DOX and apelin-13 intervention (Fig. 7B). The expression of the phosphorylated AKT and PI3K proteins with apelin-13 treatment have been activated by ML221 (Fig. 7C). Furthermore, we found ML221 could also upregulate cleaved caspase-3 and PARP expressions in $\mathrm{CoCl}_{2}$-induced apoptosis compared with apelin-13 (Fig. 7D). The phosphorylated AKT, PI3K and ERK protein expressions were significantly activated after ML221 intervention in $\mathrm{CoCl}_{2}$ compared with apelin-13 (Fig. 7E and 7F). These results suggested that apelin-13 inhibits apoptosis by binding to APJ. In brief, we plotted the mechanism of apelin-13 according to our research (Figure.S1). 


\section{Discussion}

Our current study revealed that apelin-13 has a protective effect on apoptosis and attenuates cardiotoxicity induced by DOX in vitro and in vivo. Furthermore, we demonstrated that apelin-13 attenuated apoptosis by targeting cardiomyocyte membrane via APJ. The protective effect of apelin-13 peptide on cardiotoxicity through ERK/MAPK and PI3K/AKT signaling pathway is regarded as a promising molecular mechanism. Our findings provide evidence that apelin-13 peptide inhibits apoptosis and oxidative stress, indicating its potential use for DOX-induced cardiotoxicity.

Because of cardiac toxicity caused by DOX, its clinical application is severely limited. However, the mechanism has not been illuminated. $\mathrm{CoCl}_{2}$ is a classic and effective compound used to simulate hypoxic and ischemic processes[23]. The conserved C-terminal region is essential for APJ receptor binding and functional activity[24]. Previous studies have revealed that the apelin system is an emerging pathway involved in various physiological functions[25]. The endogenous ligand of its orphan $\mathrm{G}$ proteincoupled receptor is named apelin. The $\mathrm{N}$-terminus of apelin-13 is involved in regulating its binding to the receptor APJ, while the apelin-13 C-terminus is mainly involved in regulating its biological activity[26]. Further studies showed that apelin-13 is more active than that of the longer apelin peptide. Apelin-13 is related to the formation of blood vessels, the expression of endothelial cells and the regulation of cardiac contractility and is found in peripheral tissues and the central system. In our study, we found that apelin13 can bind with APJ to exert its protective effect on primary cells, which was rescued by the application of ML221, a specific antagonist of APJ[27]. 4-oxo-6-((pyrimidin-2-ylthio)methyl)-4H-pyran-3-yl 4nitrobenzoate (ML221) is the first reported APJ antagonist, which exerts its antagonistic effect mainly by inhibition of cAMP and recruitment of $\beta$-arrestin[28]. Researches have reported that ML221 inhibited apelin-13 mediated recruitment of $\beta$-arrestin and exhibited 37-fold increased selectivity for APJ compared with the angiotensin II type 1 receptor[29]. Reporters have shown that ML221 has the potential in attenuating the activation and signaling of APJ receptor and reducing apelin-induced microvascular endothelial cell proliferation[30].

Studies have demonstrated that the endocrine system is indispensable during cardiovascular physiological and pathological processes. Many known bioactive substances secreted by heart, including ANP and BNP, have been indicated to be involved in the regulation of cardiovascular disease and sensitive as indicators of disease monitoring[31]. In vivo, apelin-13 increased heart rate and reduced college fiber formation to resist cardiotoxicity. In addition, endocrine-derived peptides play important roles in different pathological processes and engage in organ cross-talks. For instance, a vasoactive intestinal polypeptide of 28 amino acids is released by intestinal neurons[32]. Its variation is related to a variety of clinical diseases and plays vital functions in different organs. It is worth observing that some long peptides can be cleaved into smaller ones that have more consequential functions than those of long ones.

PI3K-AKT signaling pathway is a classical signaling pathway to regulates apoptosis[33]. PI $(3,4,5) \mathrm{P} 3$ is an intracellular second messenger in the cell that needs to transfer protein kinase B (AKT) to the 
membrane for activation[34]. Phosphorylated AKT mediates insulin and various growth factors to induce cell growth and promote cell survival through numerous channels[35]. ERK/MAPK is a classical signaling pathway of anti-apoptosis. When the downstream phosphorylated ERK was activated, it could inhibit the process of apoptosis. In our study, the results supported that apelin-13 resists cardiotoxicity by inhibiting apoptosis and oxidative stress by activating PI3K, AKT and ERK phosphorylation.

Our study also has some limitations. First, more clinical samples are needed to determine the exact time window of its interactions for clinical application. Second, whether the modification of apelin-13 influences its function in cardiovascular diseases needs further evaluation.

Overall, we performed a full-scale functional analysis of apelin-13 in DOX-induced cardiotoxicity and found that it can inhibit cell apoptosis in vivo and in vitro. We explored the target of apelin-13 and found that it activated the ERK/MAPK and PI3K/AKT signaling pathways to inhibit apoptosis. Therefore, our study indicates a novel insight into the mechanism of apelin-13 in inhibiting DOX-induced cardiotoxicity.

\section{Abbreviations}

DOX: Doxorubicin

$\mathrm{CoCl}_{2}$ : Cobalt chloride

PBS: Phosphate-buffered saline

CCK-8: Cell counting kit-8

JC-1: Mitochondrial membrane potential assay kit with JC-1

LDH: Lactate dehydrogenase

ELISA assay: Enzyme linked immunosorbent assay

cTnl: Cardiac troponin I

cTnT: cardiac troponin T

EF: Ejection fraction

ES: Fractional shortening

LVEDs: Left ventricular end systolic diameter

ANP: Atrial natriuretic peptide

BNP: Brain natriuretic peptide 
ML221: 4-oxo-6-((pyrimidin-2-ylthio)methyl)-4H-pyran-3-yl 4-nitrobenzoate

APELA: Apelin receptor early endogenous ligand

APJ: Apelin receptor

ERK: Extracellular signal-related kinases

JNK: Jun amino-terminal kinases

PI3K: Phosphatidylinositol 3 kinases

AKT: Protein kinase $B$

MAPK: Mitogen-activated protein kinase

Scr: Sramble

AA: amino acid

\section{Declarations}

\section{Author contributions}

Lingmei Qian designed the research. XueJun Wang, Li Zhang and MengWen Feng performed the experiments. Hao Zhang and Jia Xu analyzed the data. XueJun Wang and ZiJie Cheng wrote this manuscript. Lingmei Qian and ZiJie Cheng supervised this work.

\section{Acknowledgments}

This work was supported by National Natural Science Foundation of China $(81570209,81873540)$

Conflict of Interest: All authors read and approved the final manuscript. The authors declare no competing financial interests.

Data statement: This work described was original research that has not been published previously, and not under consideration for publication elsewhere, in whole or in part.

Data availability statement: The data that support the findings of this study are available from the corresponding author upon reasonable request.

\section{References}

1. S. Zhang,Z.-T. Li,M. Liu,J.-R. Wang,M.-Q. Xu,Z.-Y. Li, et al.; Anti-tumour activity of low molecular weight heparin doxorubicin nanoparticles for histone $\mathrm{H} 1$ high-expressive prostate cancer $\mathrm{PC}-3 \mathrm{M}$ 
cells. J Control Release. 2019.

2. K. Leemasawat,A. Phrommintikul,S.C. Chattipakorn,N. Chattipakorn; Mechanisms and potential interventions associated with the cardiotoxicity of ErbB2-targeted drugs: Insights from in vitro, in vivo, and clinical studies in breast cancer patients. Cell. Mol. Life Sci. 2020.

3. S.P. Wu,M. Tam,R.M. Vega,C.A. Perez,N.K. Gerber; Effect of Breast Irradiation on Cardiac Disease in Women Enrolled in BCIRG-001 at 10-Year Follow-Up. Int. J. Radiat. Oncol. Biol. Phys. 2017.

4. Y. Wang,T. Lei,J. Yuan,Y. Wu,X. Shen,J. Gao, et al.; GCN2 deficiency ameliorates doxorubicin-induced cardiotoxicity by decreasing cardiomyocyte apoptosis and myocardial oxidative stress. Redox Biol. 2018.

5. T.F. Lüscher; Ageing, inflammation, and oxidative stress: final common pathways of cardiovascular disease. Eur. Heart J. 2015.

6. D. Lee,S.-H. Lee,I. Noh,E. Oh,H. Ryu,J. Ha, et al.; A Helical Polypeptide-Based Potassium Ionophore Induces Endoplasmic Reticulum Stress-Mediated Apoptosis by Perturbing Ion Homeostasis. Adv Sci (Weinh). 2019.

7. J.A. Malla,R.M. Umesh,S. Yousf,S. Mane,S. Sharma,M. Lahiri, et al.; A Glutathione Activatable Ion Channel Induces Apoptosis in Cancer Cells by Depleting Intracellular Glutathione Levels. Angew. Chem. Int. Ed. Engl. 2020.

8. T. Münzel,T. Gori,R.M. Bruno,S. Taddei; Is oxidative stress a therapeutic target in cardiovascular disease? Eur. Heart J. 2010.

9. N. Ye,N. Zhang,Y. Zhang,H. Qian,B. Wu,Y. Sun; Cul4a as a New Interaction Protein of PARP1 Inhibits Oxidative Stress-Induced H9c2 Cell Apoptosis. Oxid Med Cell Longev. 2019.

10. H. Kim,G.-R. Lee,J. Kim,J.Y. Baek,Y.-J. Jo,S.-E. Hong, et al.; Sulfiredoxin inhibitor induces preferential death of cancer cells through reactive oxygen species-mediated mitochondrial damage. Free Radic. Biol. Med. 2016.

11. W. Paes,G. Leonov,T. Partridge,T. Chikata,H. Murakoshi,A. Frangou, et al.; cisContribution of proteasome-catalyzed peptide -splicing to viral targeting by CD8 T cells in HIV-1 infection. Proc. Natl. Acad. Sci. U.S.A. 2019.

12. T.A. Johnson,L. Milan-Lobo,T. Che,M. Ferwerda,E. Lambu,N.L. Mcintosh, et al.; Identification of the First Marine-Derived Opioid Receptor "Balanced" Agonist with a Signaling Profile That Resembles the Endorphins. ACS Chem Neurosci. 2017.

13. D. Brady,A. Grapputo,O. Romoli,F. Sandrelli; Insect Cecropins, Antimicrobial Peptides with Potential Therapeutic Applications. Int J Mol Sci. 2019.

14. B.S.M. Chow,T.J. Allen; Angiotensin II type 2 receptor (AT2R) in renal and cardiovascular disease. Clin. Sci. 2016.

15. C. Lugnier,A. Meyer,A. Charloux,E. Andrès,B. Gény,S. Talha; The Endocrine Function of the Heart: Physiology and Involvements of Natriuretic Peptides and Cyclic Nucleotide Phosphodiesterases in Heart Failure. J Clin Med. 2019. 
16. A. Mughal,S.T. O'rourke; Vascular effects of apelin: Mechanisms and therapeutic potential. Pharmacol. Ther. 2018.

17. A. Pauli,M.L. Norris,E. Valen,G.-L. Chew,J.A. Gagnon,S. Zimmerman, et al.; Toddler: an embryonic signal that promotes cell movement via Apelin receptors. Science. 2014.

18. J. Zhu,S. Dou,Y. Jiang,B. Bai,J. Chen,C. Wang, et al.; Apelin-36 exerts the cytoprotective effect against MPP-induced cytotoxicity in SH-SY5Y cells through PI3K/Akt/mTOR autophagy pathway. Life Sci. 2019.

19. W. Jiang,W. Hu,L. Ye,Y. Tian,R. Zhao,J. Du, et al.; Contribution of Apelin-17 to Collateral Circulation Following Cerebral Ischemic Stroke. Transl Stroke Res. 2019.

20. A. Murza,X. Sainsily,D. Coquerel,J. Côté,P. Marx,É. Besserer-Offroy, et al.; Discovery and StructureActivity Relationship of a Bioactive Fragment of ELABELA that Modulates Vascular and Cardiac Functions. J. Med. Chem. 2016.

21. D.N. Charo,M. Ho,G. Fajardo,M. Kawana,R.K. Kundu,A.Y. Sheikh, et al.; Endogenous regulation of cardiovascular function by apelin-APJ. Am. J. Physiol. Heart Circ. Physiol. 2009.

22. M. Tang,Z. Huang,X. Luo,M. Liu,L. Wang,Z. Qi, et al.; Ferritinophagy activation and sideroflexin1dependent mitochondria iron overload is involved in apelin-13-induced cardiomyocytes hypertrophy. Free Radic. Biol. Med. 2019.

23. M. Dai,P. Cui,M. Yu,J. Han,H. Li,R. Xiu; Melatonin modulates the expression of VEGF and HIF-1 alpha induced by $\mathrm{CoCl} 2$ in cultured cancer cells. J. Pineal Res. 2008.

24. K. Shin,C. Kenward,J.K. Rainey; Apelinergic System Structure and Function. Compr Physiol. 2017.

25. P. Yang,J.J. Maguire,A.P. Davenport; Apelin, Elabela/Toddler, and biased agonists as novel therapeutic agents in the cardiovascular system. Trends Pharmacol. Sci. 2015.

26. C. Read,D. Nyimanu,T.L. Williams,D.J. Huggins,P. Sulentic,R.G.C. Macrae, et al.; International Union of Basic and Clinical Pharmacology. CVII. Structure and Pharmacology of the Apelin Receptor with a Recommendation that Elabela/Toddler Is a Second Endogenous Peptide Ligand. Pharmacol. Rev. 2019.

27. L. Zhang,F. Li,X. Su,Y. Li,Y. Wang,R. Fang, et al.; Melatonin prevents lung injury by regulating apelin 13 to improve mitochondrial dysfunction. Exp. Mol. Med. 2019.

28. Q. Xiong,W. He,H. Wang,J. Zhou,Y. Zhang,J. He, et al.; Effect of the spinal apelin-APJ system on the pathogenesis of chronic constriction injury-induced neuropathic pain in rats. Mol Med Rep. 2017.

29. C. Hall,L. Ehrlich,J. Venter,A. O'brien,T. White,T. Zhou, et al.; Inhibition of the apelin/apelin receptor axis decreases cholangiocarcinoma growth. Cancer Lett. 2017.

30. Y. Ishimaru,F. Shibagaki,A. Yamamuro,Y. Yoshioka,S. Maeda; An apelin receptor antagonist prevents pathological retinal angiogenesis with ischemic retinopathy in mice. Sci Rep. 2017.

31. S.H. Reginauld,V. Cannone,S. Iyer,C. Scott,K. Bailey,J. Schaefer, et al.; Differential Regulation of ANP and BNP in Acute Decompensated Heart Failure: Deficiency of ANP. JACC Heart Fail. 2019. 
32. X. Sun,C. Guo,F. Zhao,J. Zhu,Y. Xu,Z.-Q. Liu, et al.; Vasoactive intestinal peptide stabilizes intestinal immune homeostasis through maintaining interleukin-10 expression in regulatory $B$ cells.

Theranostics. 2019.

33. Z. Lin,X.-Y. Tian,X.-X. Huang,L.-L. He,F. Xu; microRNA-186 inhibition of PI3K-AKT pathway via SPP1 inhibits chondrocyte apoptosis in mice with osteoarthritis. J. Cell. Physiol. 2019.

34. H.-P. Song,Z.-G. Chu,D.-X. Zhang,Y.-M. Dang,Q. Zhang; PI3K-AKT Pathway Protects Cardiomyocytes Against Hypoxia-Induced Apoptosis by MitoKATP-Mediated Mitochondrial Translocation of pAKT. Cell. Physiol. Biochem. 2018.

35. C. Godoy-Parejo,C. Deng,W. Liu,G. Chen; Insulin Stimulates PI3K/AKT and Cell Adhesion to Promote the Survival of Individualized Human Embryonic Stem Cells. Stem Cells. 2019.

\section{Figures}

Gly Gly Gly Trp Pro Gly Pro Gly Asn Arg Ser Gly Arg Pro Gin Val Leu $\operatorname{Arg}$

Arg

Lys Phe Arg Arg Gln Arg Pro Arg Leu Ser His Lys Gly Pro Met Pro Phe

Gin Arg Pro Arg Leu Ser His Lys Gly Pro Met Pro Phe Apelin-13

pGlu Arg Pro Arg Leu Ser His Lys Gly Pro Met Pro Phe $\left[\right.$ Pyr $^{1} \mid$ Apelin-13

\section{Figure 1}

The bioinformation of apelin. Apelin-13 is a cleaved fragment of ELA (19-32) that contains 11 amino acids. 
A.

$12 \mathrm{~h}$

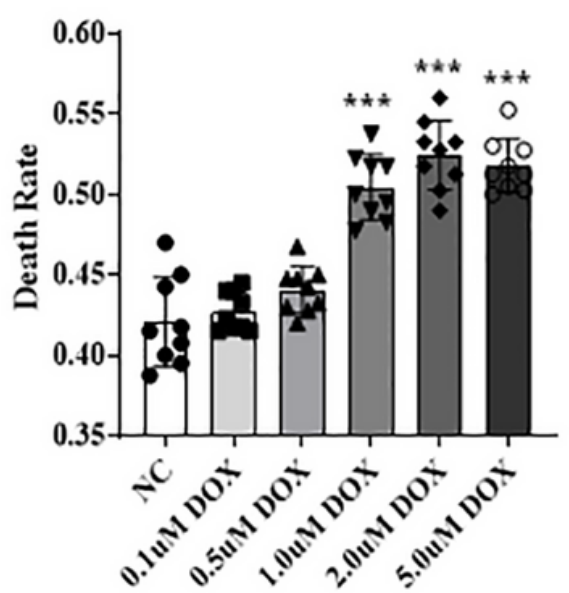

D.

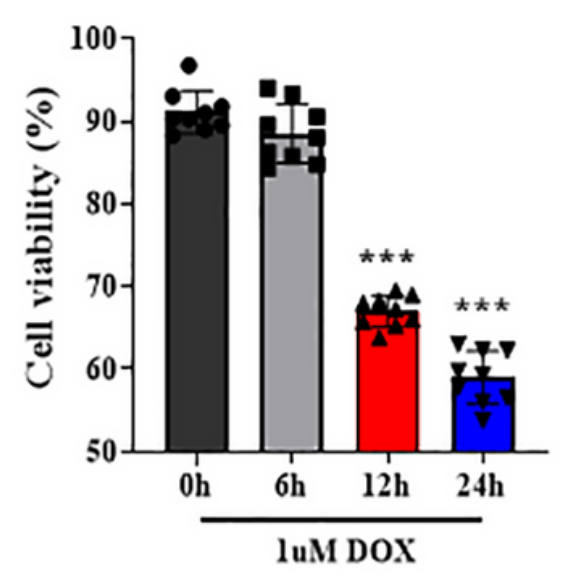

B. $12 \mathrm{~h}$

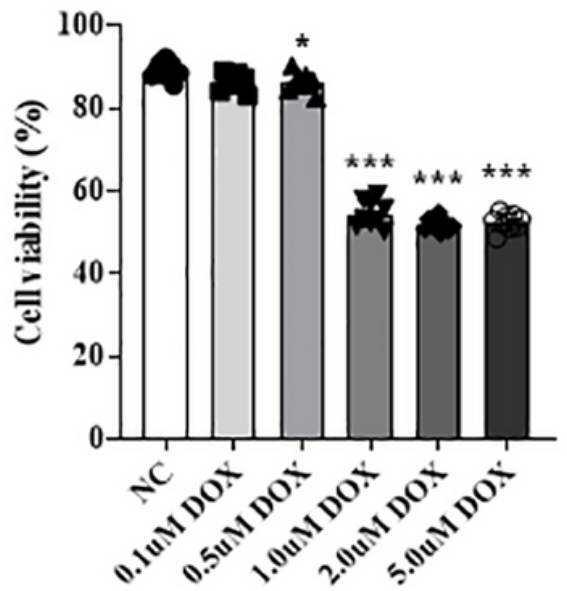

E.

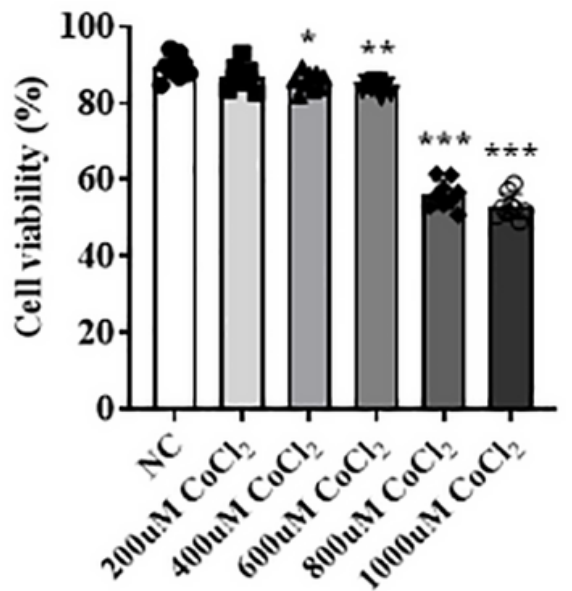

C.

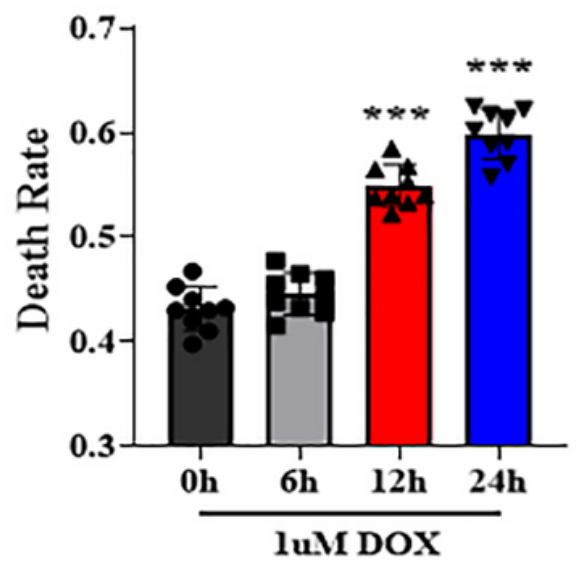

F.

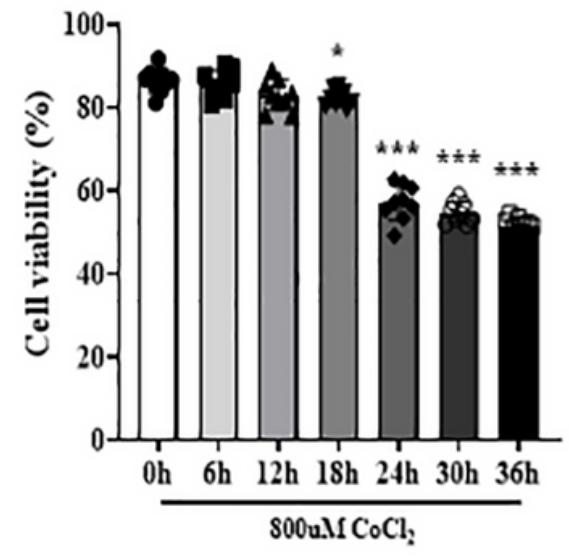

Figure 2

The effect of doxorubicin and cobalt chloride on cell viability and death. A The death rate of rat primary cells treated with $0.1,0.5,1.0,2.0$ and $5.0 \mu \mathrm{M}$ doxorubicin for $12 \mathrm{~h}$ was measured by trypan blue assay. ${ }^{\star} * * P<0.001$, one-way ANOVA, $n=9$. B The cell survival rate of DOX was measured with different concentrations for $12 \mathrm{~h}$ by CCK-8 assay. ${ }^{*} P<0.05$ and $* * * P<0.001$, one-way ANOVA, $n=9$. C The effect of $1 \mu \mathrm{M}$ doxorubicin cultured for different time points. ${ }^{*} * \mathrm{P}<0.001$, one-way ANOVA, $n=9$. D The cell viability was measured after treatment with $1 \mu \mathrm{M}$ doxorubicin for different time points. ${ }^{*} * \mathrm{P}<0.001$, one-way ANOVA, $n=9$. E The application of $200,400,600,800$ and $1000 \mu \mathrm{M} \mathrm{CoCl} 2$ in rat primary cell activity for different time points was detected by CCK-8 assay. ${ }^{*} P<0.05$, ${ }^{*} \mathrm{P}<0.01$ and $* * * P<0.001$, one-way ANOVA, $n=9$. F CCK-8 assay measured the different concentrations of $\mathrm{CoCl} 2$ for $24 \mathrm{~h} .{ }^{*} \mathrm{P}<0.05$, and ${ }^{* \star *} \mathrm{P}<0.01$, one-way ANOVA, $n=9$. 


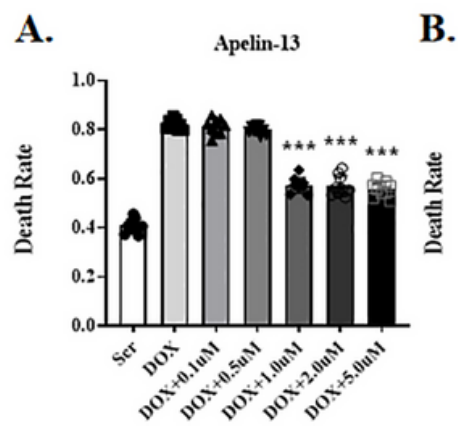

F.

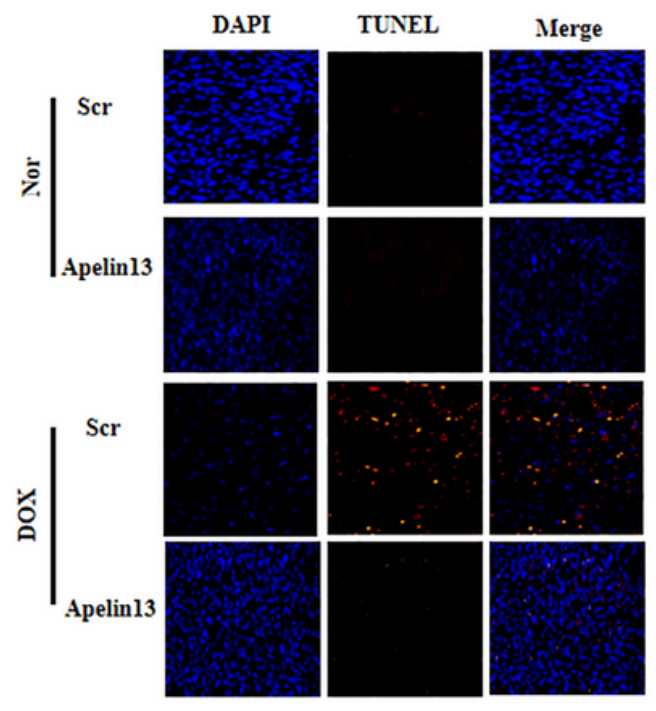

C.

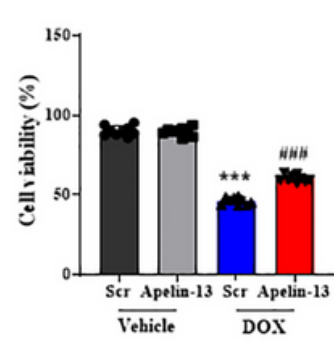

D.

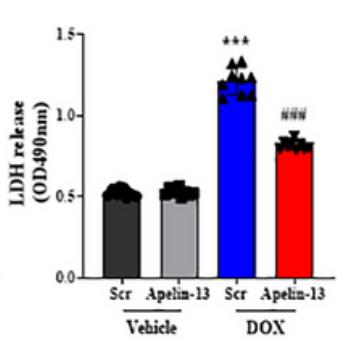

E.

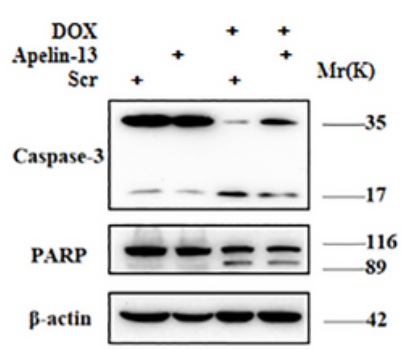

G.
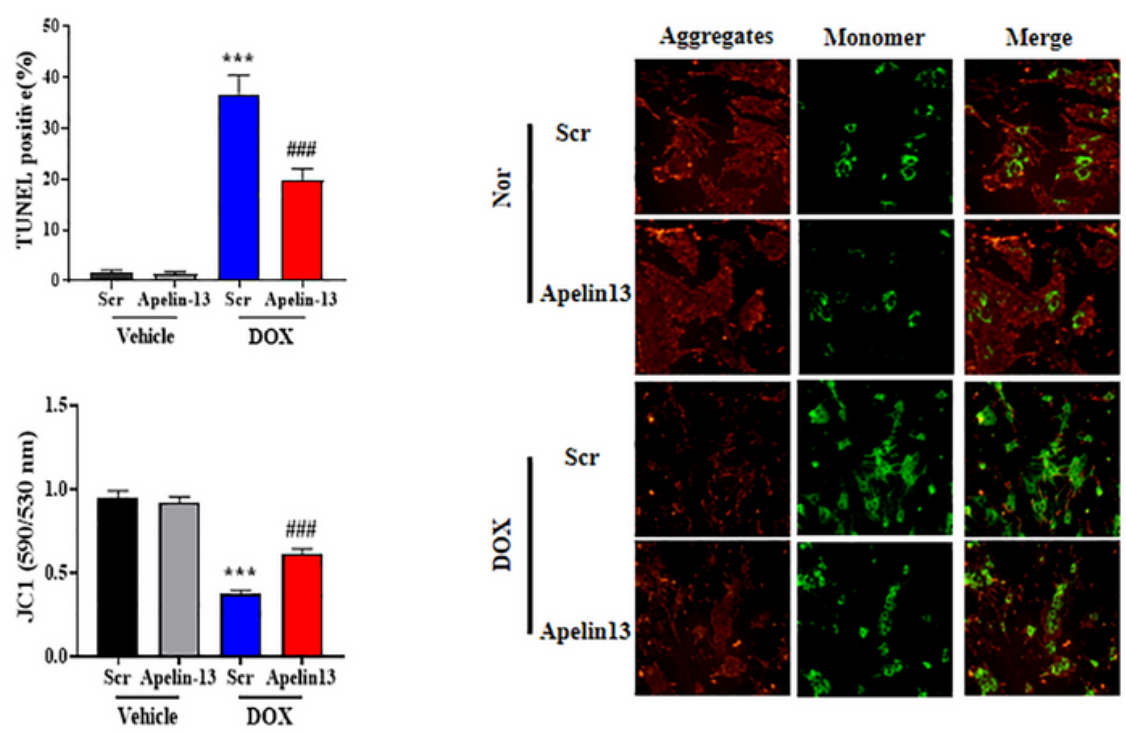

\section{Figure 3}

Apelin-13 inhibits apoptosis by attenuating doxorubicin-induced oxidative stress. A The effect of apelin$13(0.1,0.5,1.0,2.0$ and $5.0 \mu \mathrm{M})$ in DOX was measured by trypan blue assay. ***P $<0.001$, one-way ANOVA, $n=9$. B Trypan blue assay measured the effect scramble peptide $(0.1,0.5,1.0,2.0$ and 5.0 $\mu \mathrm{M})$. C Treatment with $1 \mu \mathrm{M}$ apelin-13 was measured by CCK-8 assay with DOX intervention. ***P $<0.001$, oneway ANOVA, $n=9$. D LDH release measured the effect of apelin-13 compared with DOX treated group. ${ }^{* \star * P}$ $<0.001$, one-way ANOVA, n=9. E Apoptosis markers (caspase-3 and PARP) of apelin-13 and DOX were measured by western blot. $F$ Representative photographs of apoptotic cell formation and quantification data (right). ${ }^{\star \star \star P}<0.001$, one-way ANOVA. G Changed of mitochondrial membrane permeability and quantification data (left). ${ }^{\star \star *} P<0.001$, one-way ANOVA. 
A.

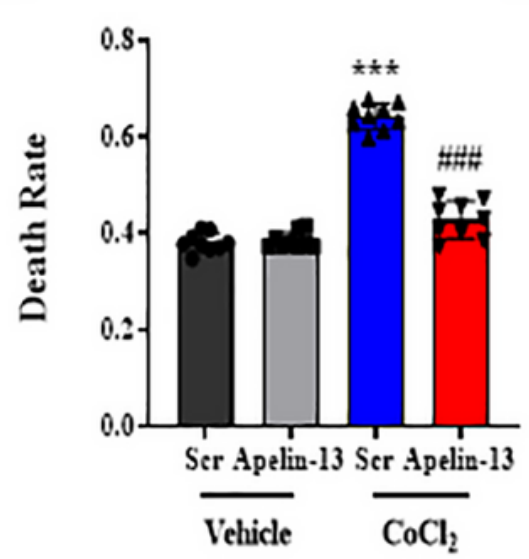

D.
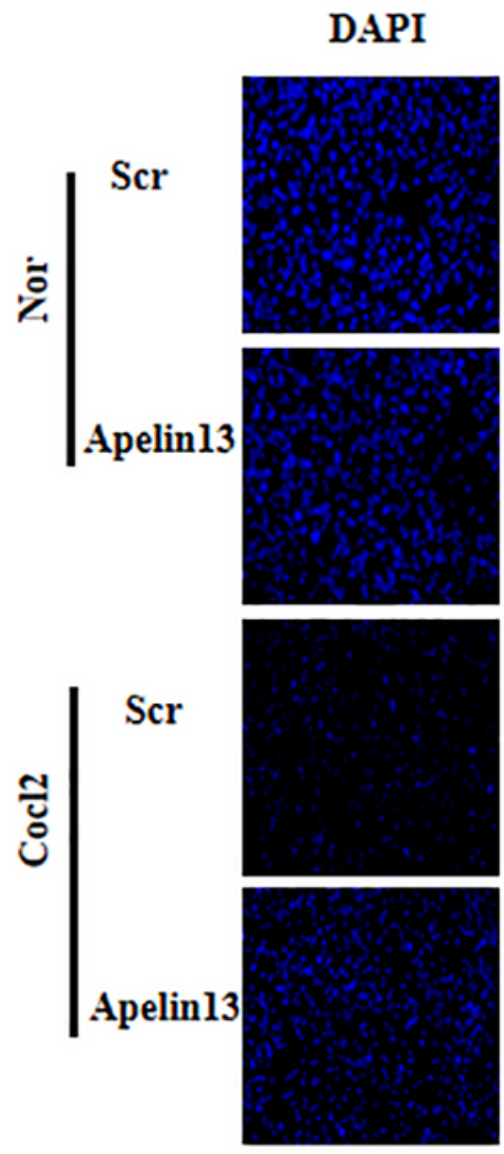

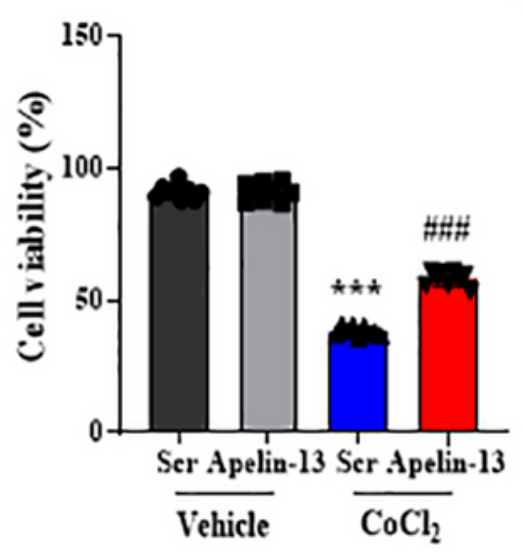

E.

C.

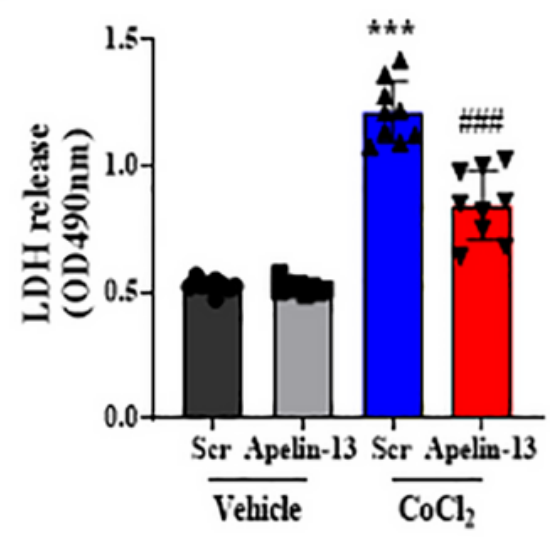

F.
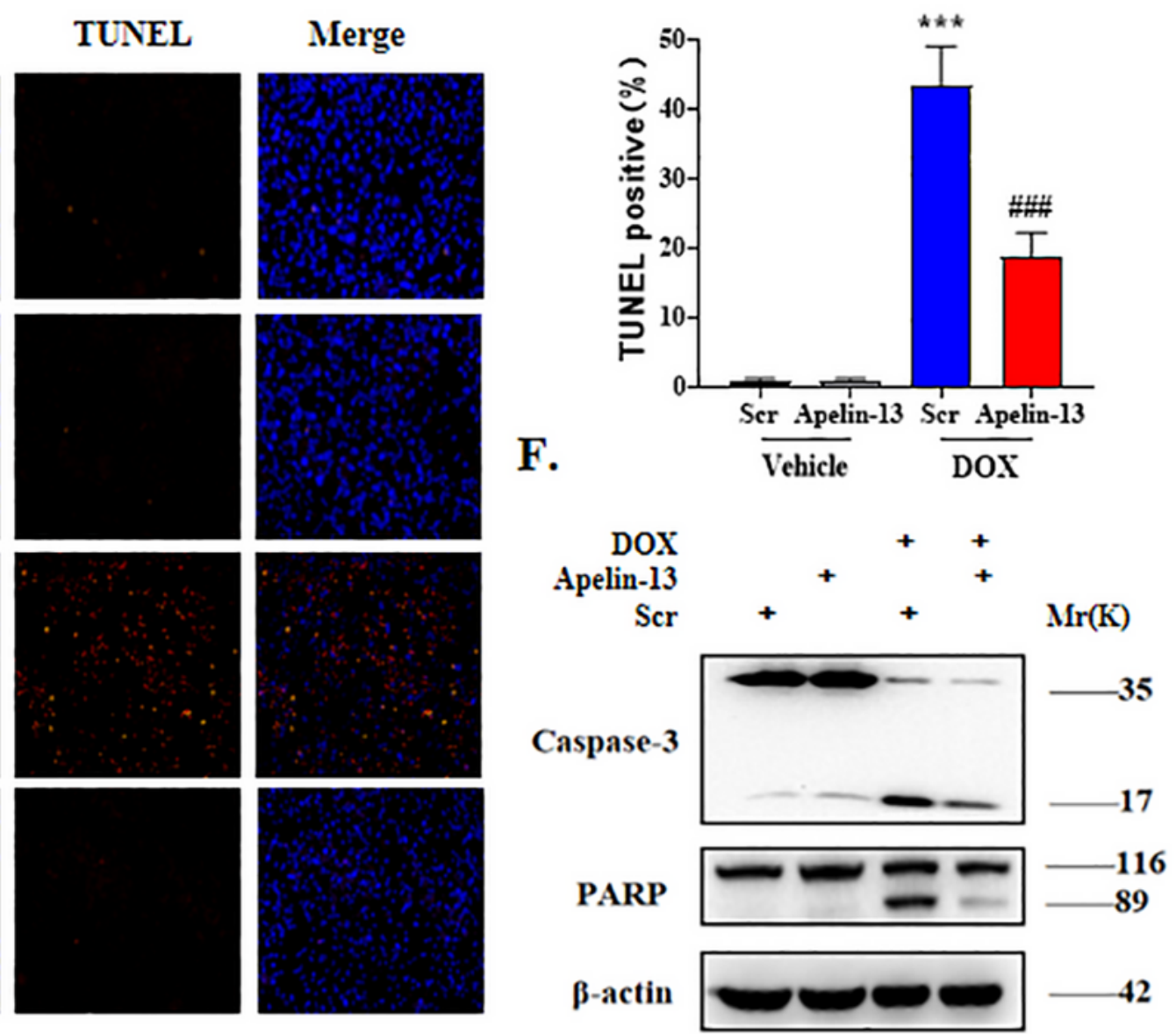

Figure 4

The effect of apelin-13 on the chemically induced hypoxic model in rat primary cells. A The cell death rate of apelin-13 with $\mathrm{CoCl} 2$ intervention was measured by trypan blue assay. ${ }^{*} * \mathrm{P}<0.001$, one-way ANOVA, $n=9$. B Cell survival rate was measured with apelin-13 treatment. *** $P<0.001$, one-way ANOVA, $n=9$. C LDH release of apelin-13 was measured with $\mathrm{CoCl} 2$ treatment. $* \star * P<0.001$, one-way ANOVA, $n=9$. D TUNEL staining assay was measured with the effect of apelin-13 in DOX model. E Quantitative data for 
TUNEL staining. ${ }^{* \star *} \mathrm{P}<0.01$, one-way ANOVA. $\mathrm{F}$ The effect of apelin-13 on CoCl2-induced apoptosis was observed by western blot analysis.

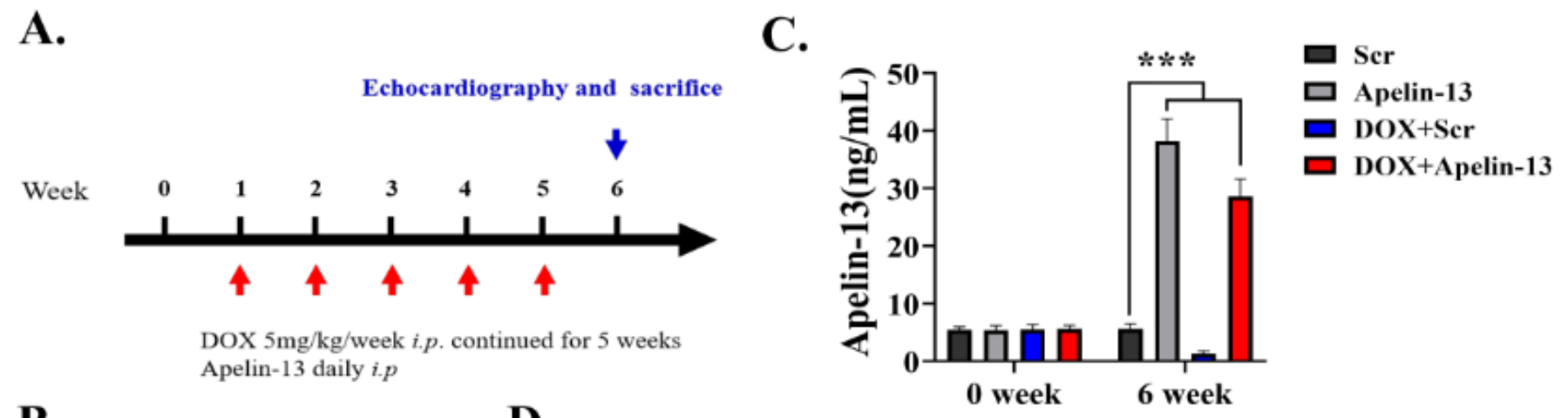

B.

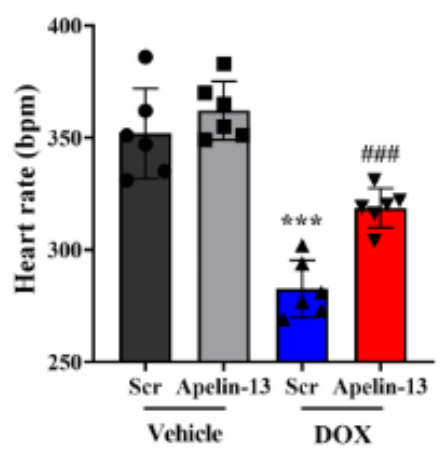

E.

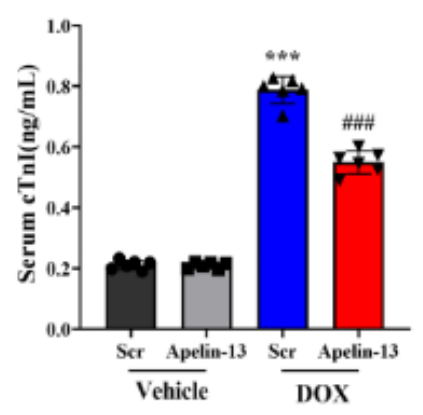

F.
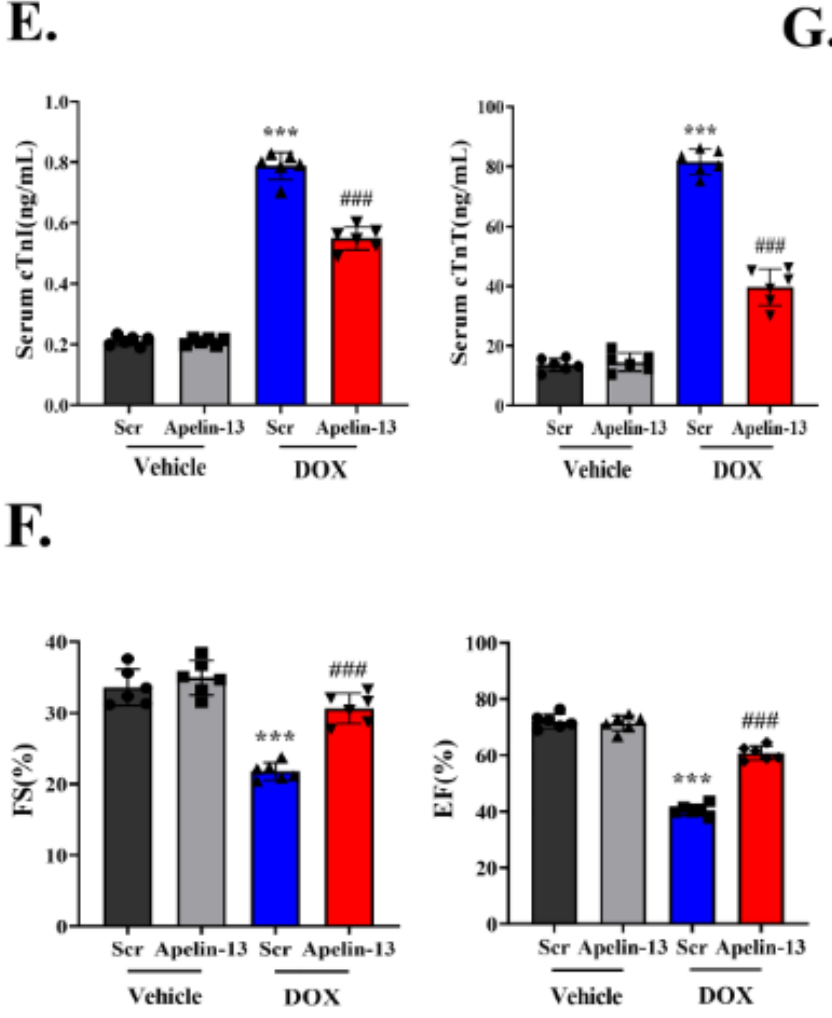

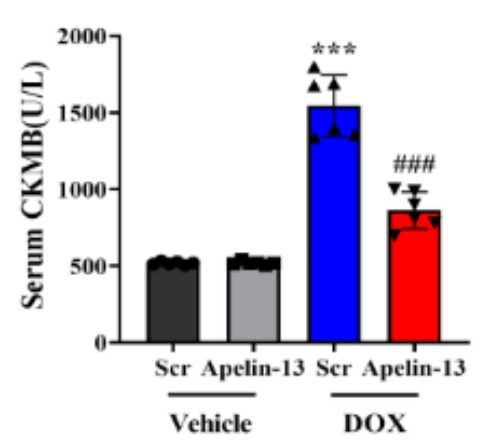

G.
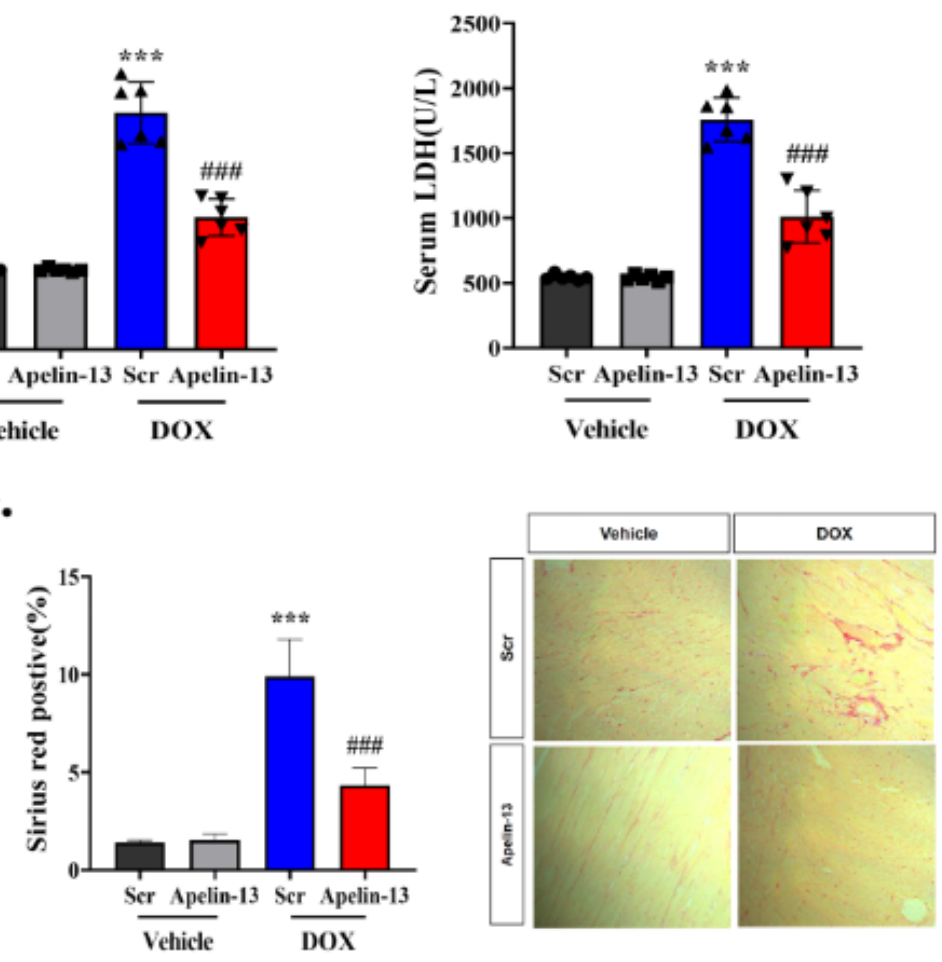

H.
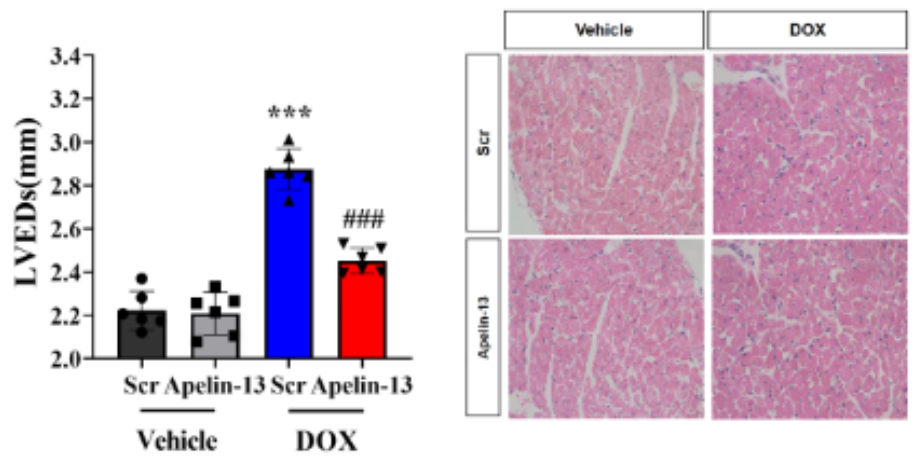

Figure 5

Apelin-13 significantly ameliorated DOX-induced heart failure in vivo. A Mice were injected with 5 $\mathrm{mg} / \mathrm{kg} /$ week doxorubicin for 5 weeks and apelin-13 was injected daily i.p, echocardiography was performed before sacrifice. B Comparison with the heart rate of the four groups. ${ }^{*} * *<<0.001$, one-way 
ANOVA, $n=6$. $C$ Expression of serum apelin-13 in four groups was measured by ELISA. ${ }^{\star \star *} P<0.001$, oneway ANOVA, $n=6$. $D$ The release of serum $C K M B$ and serum $L D H$ with apelin-13 treatment was measured by ELISA and LDH kit respectively. ${ }^{* \star *} \mathrm{P}<0.001$, one-way ANOVA, $n=6$. E Serum cTnl and cTnT expression were measured by ELISA. ${ }^{*} * * P<0.001$, one-way ANOVA, $n=6$. F EF, FS and LVEDs were quantified by echocardiography. ${ }^{*} * \mathrm{P}<0.001$, one-way ANOVA, $n=6$. G Representative photographs of Sirius red staining and quantification data (left). ${ }^{* \star} \mathrm{P}<0.001$, one-way ANOVA. H Disturbance of myocardial fiber was measured by HE staining.

A.

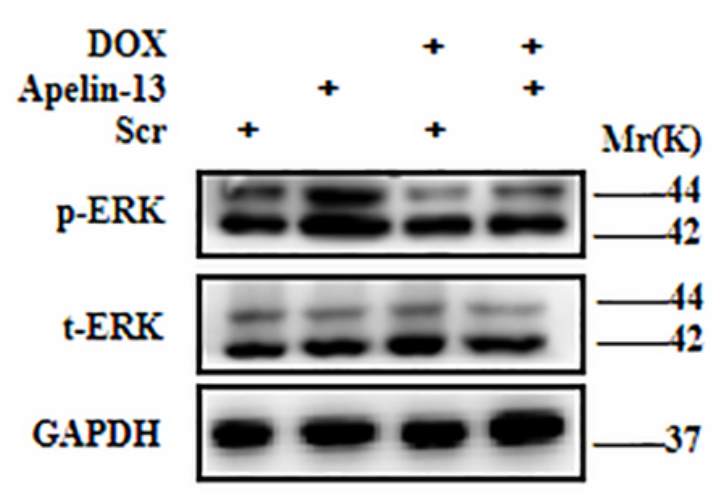

B.

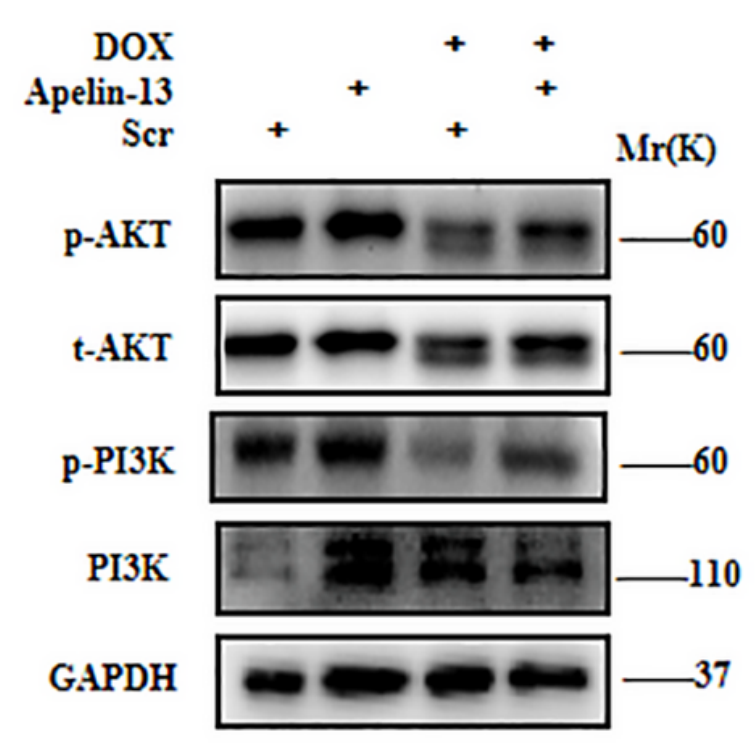

C.

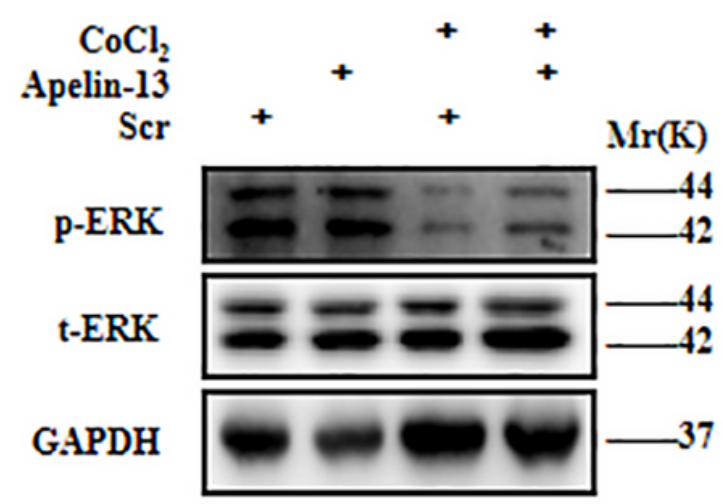

D.

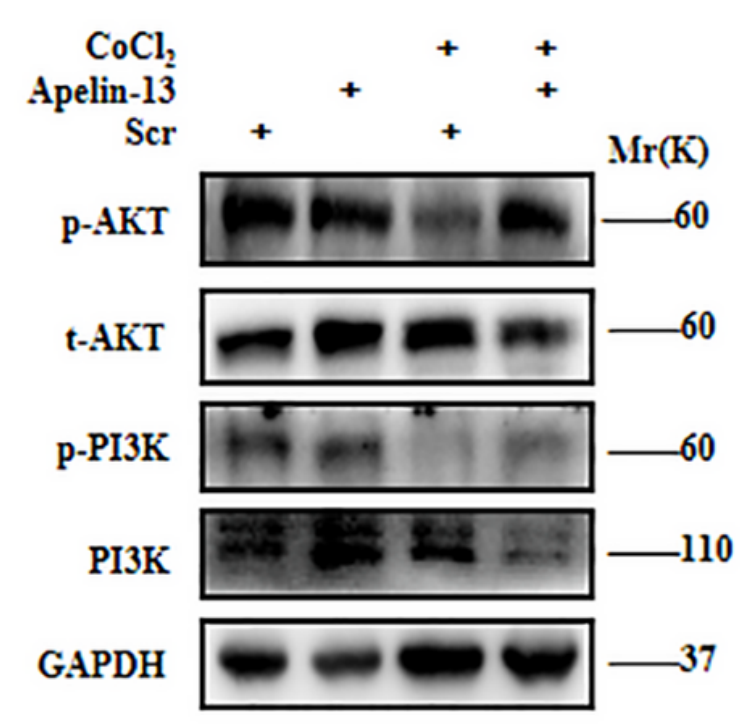

\section{Figure 6}

Apelin-13 is involved in the ERK/MAPK and PI3K/AKT signaling pathways. A ERK/MAPK signaling pathway was validated through western blot in DOX-induced model. B PI3K/AKT signaling pathway was verified by western blot in DOX-induced model. C Phosphorylated ERK protein was verified in $\mathrm{CoCl} 2-$ induced model. D The expression of phosphorylated PI3K and AKT proteins were measured by western blot with $\mathrm{CoCl} 2$ administration. 
A.

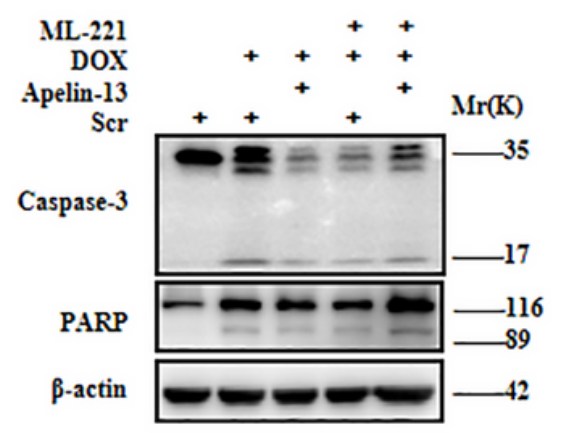

D.

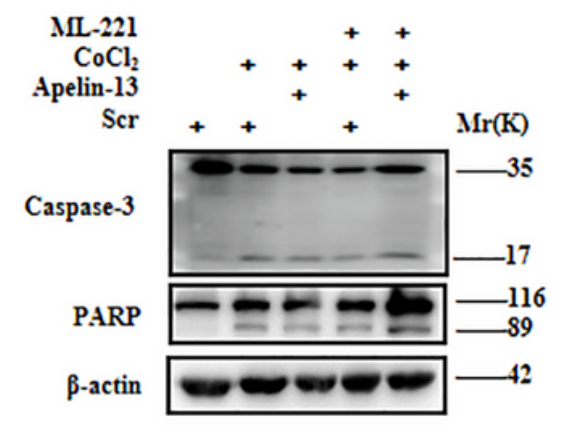

B.

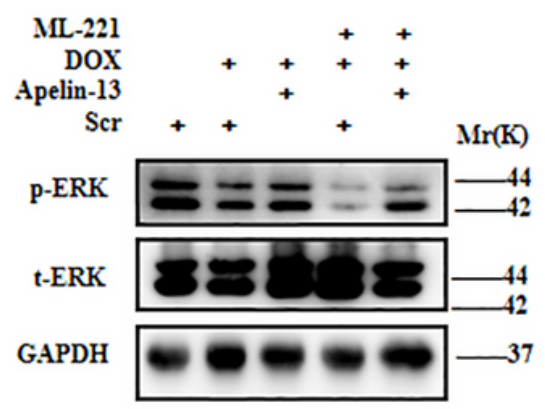

E.

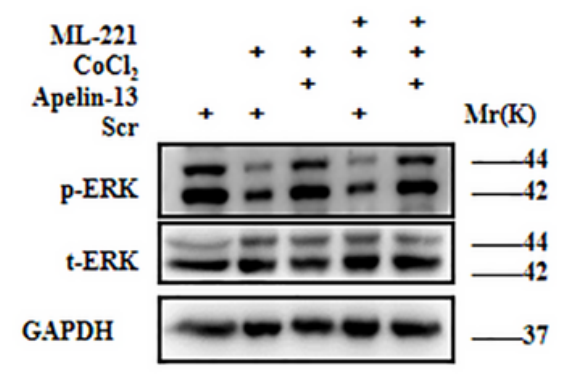

C.

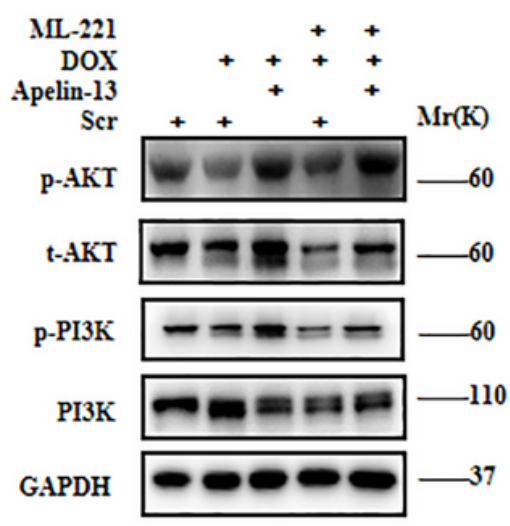

F.

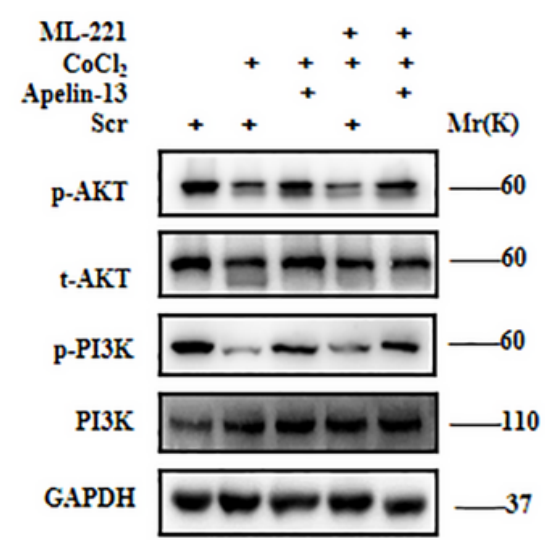

\section{Figure 7}

Apelin-13 protects cardiac function by binding APJ. A The expression of cleaved caspase-3 and PARP was validated through western blot with ML221 treatment in DOX-induced model. B The expression of ERK phosphorylated protein was measured by western blot with ML221 intervention in DOX-induced model. C The effect of ML221 in PI3K/AKT signaling pathway was verified by western blot in DOXinduced model. D The expression of cleaved caspase-3 and PARP were verified when treated with apelin13 and $\mathrm{CoCl}$ 2. E ERK/MAPK signaling pathway was verified by western blot with ML221 treatment in $\mathrm{CoCl} 2$-induced model. $\mathrm{F} \mathrm{p}$-PI3K and p-AKT protein expressions were validated by western blot when treated with ML221 and $\mathrm{CoCl} 2$.

\section{Supplementary Files}

This is a list of supplementary files associated with this preprint. Click to download.

- Supplemetalfigure.1.tif 